\title{
Familial combined hypolipidemia due to mutations in the ANGPTL3 gene
}

The role of ANGPTL3 in lipoprotein metabolism emerged from studies in a mutant mouse strain characterized by severe hypotriglyceridemia and carrying a loss-of-function (LOF) mutation of the ANGPTL3 gene. ANGPTL3 was found to inhibit lipoprotein lipase and endothelial lipase. Genome-wide association studies in humans demonstrated the association of ANGPTL3 variants with plasma triglyceride levels and LOF mutations of ANGPTL3 were found in hypotriglyceridemic subjects in population studies. Recently, individuals originally classified as affected by familial hypobetalipoproteinemia were found to be homozygotes/compound heterozygotes for rare LOF mutations of ANGPTL3. They show a striking reduction of all lipoprotein classes (VLDL, LDL and HDL), a condition defined as familial combined hypolipidemia. This disorder, transmitted as a recessive trait, does not seem to be associated with specific clinical manifestations, such as premature atherosclerosis or fatty liver disease.

KEYWORDS: ANGPTL3 ANGPTL8 endothelial lipase familial combined hypolipidemia HDL LDL lipoprotein lipase

\section{ANGPTL3 \& plasma lipoprotein metabolism in mice \& humans}

ANGPTLs represent a family of secreted glycoproteins with high homology to angiopoietins, the key regulators of angiogenesis. However, ANGPTLs cannot bind the angiopoietin receptors (TIE1 and TIE2) specifically expressed on endothelial cells [1]. ANGPTLs share two common domains with angiopoietins, a coiledcoil domain at the $\mathrm{N}$-terminal region and a fibrinogen-like domain at the C-terminal, which are connected by a linker region. Two members of the ANGPTL family (ANGPTL3 and ANGPTL4) have received great attention in the last few years due to their effects on lipoprotein metabolism in mice and humans ([2-5] for review).

The first observation which demonstrated a link between ANGPTL3 and lipoprotein metabolism was made by Koishi et al. who investigated a mouse strain (KK/San or KK/Snk) derived from the colony of KK mice characterized by obesity, diabetes and hypertriglyceridemia [6]. The KK/San mice, despite maintaining the phenotype of obesity and diabetes, had a marked decrease $(>90 \%)$ of plasma triglyceride (TG) as compared with the original hyperlipidemic KK mice. This hypotriglyceridemia was associated not only with a reduction in pre- $\beta$ lipoproteins (VLDL), but also in the $\beta$ - and $\alpha$-lipoproteins (LDL and HDL, respectively). The hypotriglyceridemia of KK/San mice was found to be inherited as a recessive trait. Using positional cloning, Koishi et al. identified the hypolipidemia locus (located in the middle of chromosome 4) in the gene encoding ANGPTL3 [6]. KK/San mice were homozygous for a 4-bp insertion in exon 7, which introduced a stop codon at position 347, predicted to result in the truncation of the ANGPTL3 protein and expected to be associated with a complete ANGPTL3 deficiency. Koishi et al. also showed that treatment with recombinant ANGPTL3 or adenovirus-mediated overproduction of ANGPTL3-elevated plasma TG and total cholesterol (TC) [6]. Subsequent studies showed that ANGPTL3 increased VLDL-TG levels by inhibiting lipoprotein lipase (LPL) activity $[7,8]$ and that the plasma TG level was markedly reduced in ANGPTL3 null mice (Angptl3-- ${ }^{--}$[9].

An extensive investigation of Angptl3 $3^{--}$mice fed a normal diet or a high-fat diet revealed a great reduction of plasma lipid levels (73 and $55 \%$ in TG and 40 and $45 \%$ in TC, respectively) [10]. This confirmed that complete ANGPTL3 deficiency had a profound effect not only on TG-containing lipoproteins (VLDL), but also on cholesterol-carrying lipoproteins such as

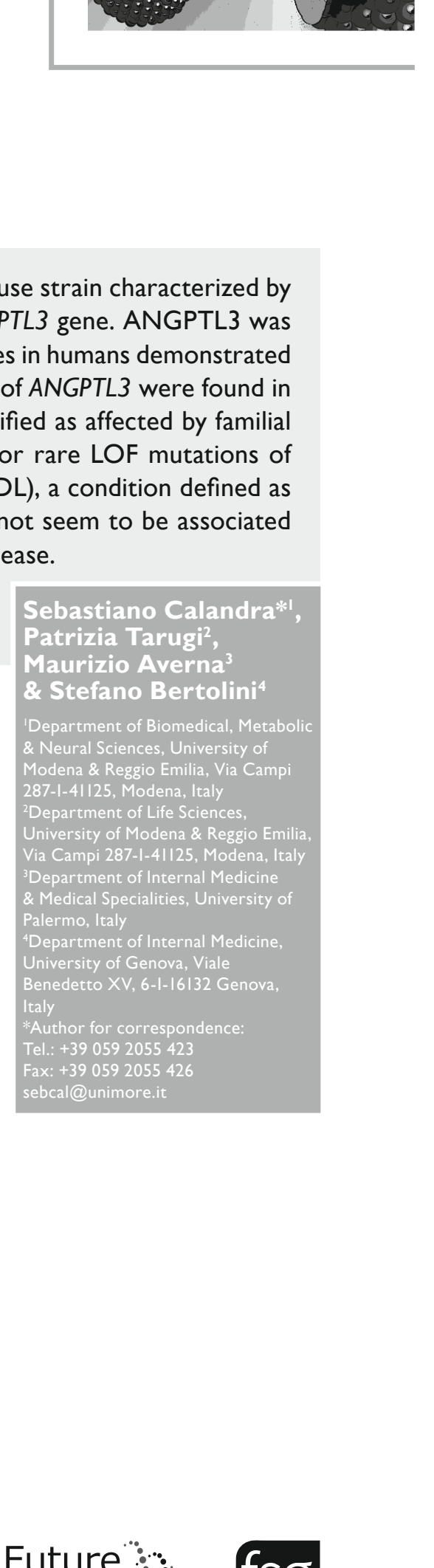


LDL and HDL. Postheparin LPL and hepatic lipase activities were significantly increased in Angptl3-/ (1.57- and 1.42-fold, respectively). Furthermore, in vivo hepatic TG secretion rate in Angptl3 ${ }^{--}$injected with Triton WR1339 was found to be lower than in control mice, although the difference did not reach the level of significance [10]. In conclusion, Angpt/3-1- had a combined hypolipidemia phenotype associated with enhanced LPL and hepatic lipase activity [10]. Unfortunately, this study did not provide data on the plasma lipids in Angtpl3+/- mice.

In humans, the ANGPTL3 gene is located on chromosome 1 (1p31.1-p22.3), where it encodes a 460 amino acid protein that is produced exclusively by the liver. ANGPTL3 consists of a signal peptide, an $\mathrm{N}$-terminal segment containing coiled-coil domains and a C-terminal fibrinogen-like domain, which are connected with a linker region. ANGPTL3 is susceptible to proteolytic cleavage at specific sites in the interlinker region by the action of several hepatic pro-protein convertases (PCs): Furin, PCSK2, PCSK4, PACE4, PCSK5 and PCSK7 (Figure I) [2]. The cleaved N-terminal of ANGPTL3 is more efficient at inhibiting LPL than full-length ANGPTL3 and the deletion of the N-segment leads to the total loss of the inhibitory activity of the protein $[7,8]$. Recently, a specific amino acid sequence of the N-terminal of ANGPTL3 has been identified as the domain that mediates

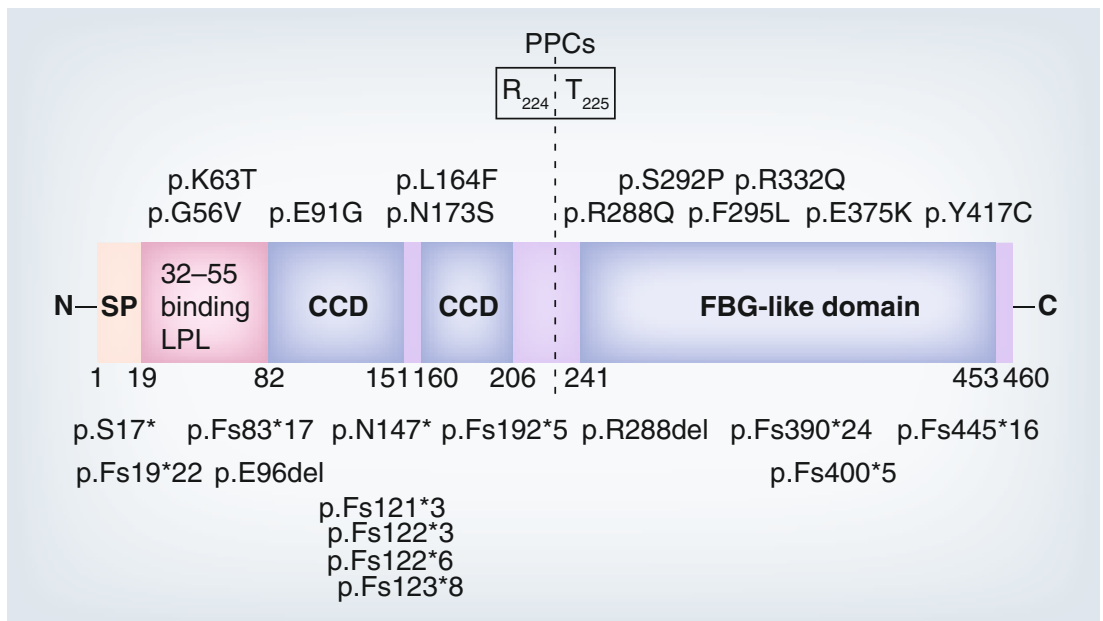

Figure 1. Structure of human ANGPTL3 protein. The protein contains a SP followed by an $\alpha$-helical region to form a CCD at the N-terminus and a FBG at the $\mathrm{C}$-terminus. These domains are separated by a linker region that is cleaved by the action of PPCs. Some missense mutations found in hypolipidemic subjects, reported to prevent the secretion or affect the function of ANGPTL3 in vitro, are indicated above the protein structure. Mutations producing truncated proteins or single amino acid deletions are shown below the protein structure. CCD: Coiled-coil domain; FBG: Fibrinogen globular domain; PPC: Pro-protein convertase; SP: Signal peptide. the interaction between ANGPTL3 and LPL, and the subsequent inactivation of LPL. This region corresponds to amino acids E32-H55 (Figure I); a mouse antibody against this region inhibited the binding of ANGPTL3 to LPL and neutralized ANGPTL3-mediated inhibition of LPL [11]. The mechanism underlying the inhibition of LPL by ANGPTL3 appears to be manifold. By using enzyme kinetic analysis with purified recombinant proteins, Shan et al. found that ANGPTL3 reduced LPL catalytic activity (i.e., the activity of the dimeric form of LPL), but did not significantly alter the LPL selfinactivation rate (i.e., the process leading to the irreversible disruption of the dimeric form of the enzyme) [12]. An alternative or complementary mechanism of inhibition of LPL has emerged from in vitro cell-based studies that investigated the interactions among ANGPTL3, PCs and LPL. These studies demonstrated that LPL is inactivated by cleavage by PCs and that ANGPTL3 is a potent inhibitor of LPL in physiological concentrations and in the presence of cells by enhancing the cleavage of LPL by PCs such as furin and PACE-4.

By enhancing LPL cleavage, ANGPTL3 dissociates LPL from the cell surface, thus inhibiting both the catalytic and noncatalytic functions of this enzyme [13]. One interesting question is whether ANGPTL3 exerts its inhibitory effect on the LPL that is bound to TG-rich lipoproteins (chylomicrons and VLDL) or to GPIHBP1, the platform for LPL-processing of TG-rich lipoproteins [14]. Recently, Nilsson et al. have investigated the effects of some lipoproteins (chylomicrons, VLDL and LDL) on the inactivation of LPL in vitro by the human $\mathrm{N}$-terminal fragment of ANGPTL3 [15]. They found that the presence of chylomicrons and VLDL protected LPL against inactivation by ANGPTL3. On the other hand, it has been shown that ANGPTL3 inhibits heparin-bound LPL but not LPL bound to GPIHBP1 [16]. So it appears that ANGPTL3 exerts its inhibitory effect predominantly on nonstablized LPL (Figure 2).

In addition to its role in the inhibition of LPL, ANGPTL3 is capable of inhibiting endothelial lipase (EL). Shimamura et al. found that Angpt $13^{-1-}$ had low plasma HDL cholesterol (HDL-C) levels accompanied by increased phospholipase activity [17], indicating that the activity of EL (which acts as a phospholipase) is elevated in these mice and that ANGPTL3 acts as an endogenous inhibitor of EL. Interestingly, 


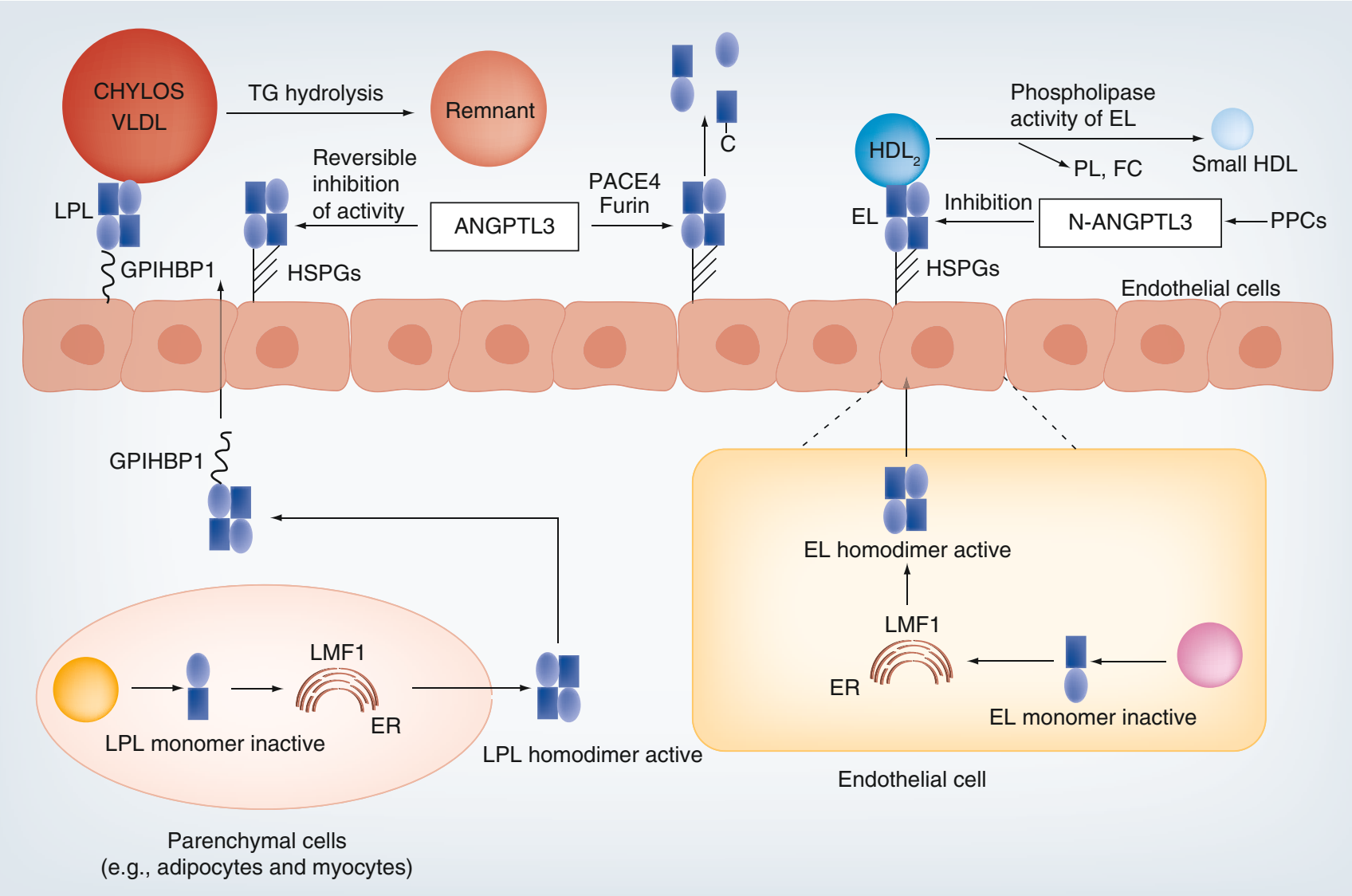

Figure 2. Model of interactions of ANGPTL3 with lipoprotein lipase and endothelial lipase in peripheral tissues.

LPL synthezised in adipose tissue is secreted as a homodimer by the action of LMF1 and then transported to the luminal side of the capillary endothelial cells by transendocytosis mediated by GPIHBP1. ANGPTL3 binds to unstabilized LPL (i.e., LPL that is not bound to chylomicrons/VLDL on the GPIHBP1 molecular platform). ANGPTL3 may induce the reversible inhibition of LPL or the irreversibile degradation of LPL by PPCS (PACE4 and Furin). The N-terminal fragment of ANGPTL3 (N-ANGPTL3) cleaved by PPCs and also inhibits EL. CHYLOS: Chylomicron; EL: Endothelial lipase; ER: Endoplasmic reticulum; FC: Free cholesterol; HSPG: Heparan sulfate proteoglycan; LPL: Lipoprotein lipase; PL: Phospholipid; PPC: Pro-protein convertase; TG: Triglyceride.

the inhibition of PCs, which are responsible for the activation of ANGPTL3 (as previously mentioned) attenuated the inhibitory effect of ANGPTL3 on EL [18].

Thus, PCs that regulate EL activity directly through the cleavage of the EL protein [19] also affect this enzyme indirectly by modulating the ANGPTL3 level in the liver. In this context, the changes of HDL, seen in Angptl3 ${ }^{-1}$ mice due to unrestrained EL activity, are expected to bear some similarity with those observed in mice overexpressing EL. Indeed, overexpression of EL in mice by a recombinant adenovirus dramatically lowered HDL-C and ApoA-I [20].

Activation of ANGPTL3 by ANGPTL8, a new member of the ANGPTL family

Recently, the activity of ANGPTL3 on plasma lipoprotein has been linked to a new member of the ANGPTL family, designated ANGPTL8 [21], which had been previously designated with various terms (TD26, hepatocellular carcinomaassociated gene, C19orf80, refeeding-induced fat and liver or lipasin) [22,23]. Zhang was the first to show that lipasin (ANGPTL8) shared homology with the N-terminal domain of ANGPTL3, which is needed for inhibition of LPL; recombinant human lipasin inhibited LPL in vitro and adenovirus-mediated injection of lipasin increased mouse serum TGs [23].

The role of ANGPTL8 has been extensively investigated by Quagliarini et al. who demonstrated that ANGPTL8 is a plasma protein whose expression is modulated by fasting and refeeding [21]. They found that the expression of human ANGPTL8 in the livers of mice was associated with a significant increase in plasma levels of TG and nonesterified fatty acids (NEFA). By contrast, the expression of human ANGPTL3 did not induce elevation of TG or NEFA, while the coexpression of ANGPTL8 and ANGPTL3 induced a striking increase of both TG and NEFA, thus suggesting 
a concerted action of these proteins. In cultured hepatocytes, ANGPTL8 expression increased the appearance of $\mathrm{N}$-terminal ANGPTL3 in the medium, suggesting that ANGPTL8 may activate ANGPTL3 by facilitating its cleavage. Consistent with this hypothesis, the expression of ANGPTL8 in Angptl3 ${ }^{-/-}$mice failed to promote hypertriglyceridemia. Therefore, in this new scenario, ANGPTL8 would be required to ensure full biological activity of ANGPTL3 on plasma lipoproteins (Figure 3). It has been proposed that ANGPTL8 activates ANGPTL3 by binding to the N-terminal domain of the fulllength ANGPTL3 and promoting its cleavage. After cleavage, ANGPTL8 remains bound to ANGPTL3 and forms an active complex circulating in plasma (Figure 3) [21]. Thus, ANGPTL8 acts to increase plasma TG levels and this requires the presence of ANGPTL3. This is consistent with the observation that Angptlo-/- mice have markedly decreased TG levels compared with wild-type mice [24].

\section{Plasma levels of ANGPTL3 \& correlation with plasma lipids}

Using an antibody sandwich ELISA system, Shimamura et al. found that the concentration of ANGPTL3 in the plasma of healthy Japanese subjects (mean age: $33.6 \pm 8.4$ years; male/female: 45/42) in overnight fasting conditions ranged from 50 to $800 \mathrm{ng} / \mathrm{ml}$ (mean value: $470 \pm 122 \mathrm{ng} / \mathrm{ml}$ ) [17]. Plasma ANGPTL3 correlated strongly with plasma HDL-C $(\mathrm{r}=0.500 ; \mathrm{p}<0.001)$ and HDL-phospolipid levels $(\mathrm{r}=0.286 ; \mathrm{p}=0.007)$, but not with plasma TC or TG levels [17]. Hatsuda et al.,

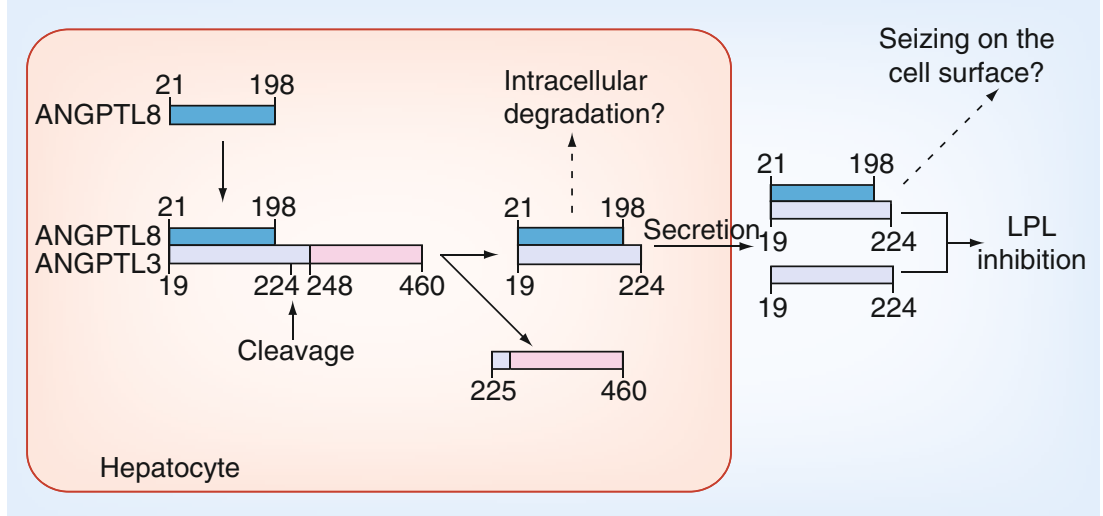

Figure 3. Model of interaction of ANGPTL8 with ANGPTL3 in hepatocytes. ANGPTL8-ANGPTL3 interaction facilitates the proteolytic cleavage of ANGPTL3. ANGPTL8 remains attached to the N-terminal fragment of ANGPTL3, forming a complex that is secreted. Both the ANGPTL3-ANGPTL8 complex and the free $\mathrm{N}$-terminal fragment of ANGPTL3 inhibit LPL. LPL: Lipoprotein lipase. using a commercially available kit assay, showed that in 381 healthy Japanese subjects, the mean plasma level of ANGPTL3 was $764 \pm 291 \mathrm{ng} / \mathrm{ml}$, with no difference between males and females [25]. By simple linear regression analysis, plasma ANGPTL3 levels did not correlate with age, BMI or plasma lipid parameters (TC, HDL-C and TG), fasting blood glucose, log-transformed insulin or the homeostasis model assessment of insulin resistance. Robciuc et al., using a noncompetitive ELISA assay, found that the plasma/serum concentration of ANGTPL3 was $347.8 \pm 159.4$ and $388.6 \pm 204.1 \mathrm{ng} / \mathrm{ml}$ in healthy Finnish middle-aged males $(n=125)$ and females $(\mathrm{n}=125)$, respectively [26]. Bivariate correlations revealed a positive association of serum ANGPTL3 with age $(r=0.292$; $p<0.001)$, plasma HDL-C $(r=0.224 ; \mathrm{p}<0.001)$ and ApoA-I $(r=0.144 ; \mathrm{p}=0.023)$, and $\mathrm{a}$ negative correlation with plasma TG $(r=-0.182$; $\mathrm{p}=0.004)$. However, the correlation with plasma TG was completely lost when HDL-C and ApoA-I were used as control variables [26]. A strong correlation between plasma ANGPTL3 and HDL-C was also documented by Moon et al. in a group of Japanese subjects that also included individuals with hyperalphalipoproteinemia (HDL-C level $>90 \mathrm{mg} / \mathrm{dl}$; $>2.33 \mathrm{mmol} / \mathrm{l}$ ) [27]. Overall, it seems that that there is a positive correlation between plasma ANGPTL3 and plasma HDL in accordance with the studies in mice [17]. This relationship is indirectly supported by the observation that among Japanese individuals with elevated HDL-C $(>90 \mathrm{mg} / \mathrm{dl}$; $>2.33 \mathrm{mmol} / \mathrm{l}$ ), the prevalence of high plasma ANGTPL3 levels (>362 ng/ml) was found to be tenfold higher (44\%) compared with that $(4.5 \%)$ of low plasma CETP $(<0.39 \mu \mathrm{g} / \mathrm{ml}$; normal values $1.29 \pm 0.90 \mu \mathrm{g} / \mathrm{ml}$ ), a condition known to be associated with elevated plasma HDL-C [27]. The plasma levels of ANGPTL3 have also been associated with preclinical atherosclerosis. Hatsuda et al. found a positive association between plasma ANGPTL3 and carotid and femoral artery intima-medial thickness in 381 healthy volunteers after adjustment for other cardiovascular risk factors such as age, blood pressure and plasma glucose and lipid levels [25].

\section{ANGPTL3 gene variants \& plasma lipids in population studies}

Recently, several genetic approaches have unequivocally proved the key role of ANGPTL3 in human lipoprotein metabolism. Genomewide association studies (GWAS) have been 
used as unbiased tools to identify novel genes associated with lipid traits in a population. Among the loci associated with plasma TG, a locus was identified on chromosome $1 \mathrm{p} 31$ near the ANGPTL3 gene [28]. The intergenic single nucleotide polymorphism rs12130333 (minor allele frequency $[\mathrm{MAF}]=0.22$ ) was associated with a reduction in plasma TG [28]. This observation was confirmed in a subsequent study and a meta-analysis [29], which showed that the rare allele of another single nucleotide polymorphism (rs2131925, MAF $=0.32)$ near the ANGPTL3 gene had an effect size on plasma TG of $-4.95 \mathrm{mg} / \mathrm{dl}$.

GWAS prompted the population-based resequencing of the coding region of the ANGPTL3 gene, which led to the identification of multiple rare nonsynonymous (NS) sequence variants that were associated with reduced plasma TG levels in individuals of the DHS and ARIC study [30]. A total of 35 rare NS amino acid variants were identified in ANGTPL 3 and an excess of these variants ( 14 vs $5 ; \mathrm{p}=0.064$ ) were found in the lower quartile of plasma TG levels (Tables I \& 2). All sequence variants expected to be loss-of-function (LOF) alleles (which impaired the secretion of the mutant ANGPTL3 from the cells in vitro or produced truncated ANGPTL3 proteins) were in the lower quartile of TG levels of the reference population. In addition, the variant Met259Thr (M259T), a relatively frequent NS variant in African-Americans (MAF $=0.05$ ) was found to be associated with significantly lower plasma TG, with a clear gene-dosage effect. In vitro studies indicated that the ANGPTL3-259T variant, as well as some other rare NS variants found in the low TG group, failed to suppress LPL activity in vitro, as compared with the wild type $A N G P T L 3$ [30]. Interestingly, the rare LOF variants in $A N G P T L 3$, found in individuals with low plasma TG, were not associated with significant changes in the levels of LDL cholesterol (LDL-C) and HDL-C. No changes of other metabolic parameters were observed in carriers of common or rare LOF variants, apart from a low plasma level of blood glucose found in carriers of the DHS population. Regrettably, no data were given on the plasma level of ANGPTL3 in carriers of these variants [30].

\section{ANGPTL3 \& familial combined hypolipidemia}

The compelling evidence of the role of LOF mutations of ANGPTL3 as the cause of hypolipidemia in humans emerged from the genomic investigation of a large family, originally classified as familial hypobetalipoproteinemia (FHBL) by Schonfeld's group in 1998 [31]. FHBL includes a heterogeneous group of autosomal codominant disorders characterized by plasma levels of TC, LDL-C and ApoB below the fifth percentile of the population distribution [32]. Approximately $50 \%$ of FHBL subjects identified in clinical settings are carriers of mutations in the $A P O B$ gene ( $A P O B$-linked FHBL), which either prevent the complete translation of $A P O B$ mRNA (resulting in the production of truncated ApoBs of various length) or result in missense mutations that cause an accelerated intracellular degradation of the mutant APOB [32]. A total of $2 \%$ of FHBL subjects are carriers of LOF mutations in the PCSK9 gene (PCSK9-linked FHBL), while in others, the genes responsible for the disorder are unknown ('orphan FHBL') [32]. In the FHBL family described by Schonfeld's group (indicated as family 'F'), the $A P O B$ gene had been excluded by cosegregation analysis [31]. By using the strategy of exome sequencing, Musunuru et al. demonstrated that in this family, four hypocholesterolemic siblings were compound heterozygotes for two LOF mutations of ANGPTL3 (p.E129*/p.S17*), which are expected to cause a complete deficiency of ANGPTL3 (Table 2 \& Figure I) [33]. Interestingly, these subjects, besides extremely low levels of TC and LDL-C $(1.41 \pm 0.07$ and $0.84 \pm 0.09 \mathrm{mmol} / \mathrm{l}$, respectively), had very low levels of TG and HDL-C $(0.23 \pm 0.03$ and $0.46 \pm 0.07 \mathrm{mmol} / \mathrm{l}$, respectively). The discovery of this lipid disorder, designated familial combined hypolipidemia, paved the way to the resequencing of the ANGPTL3 gene in other putative FHBL subjects with a combined hypolipidemia phenotype, in whom no mutations in $A P O B$ or PCSK9 genes had been identified. The present authors resequenced the ANGPTL3 gene in four individuals (belonging to three kindreds) with a plasma lipid profile comparable to that of family ' $F$ ' (TC: $1.76 \pm 0.30$; TG: $0.49 \pm 0.13$; LDL-C: $0.97 \pm 0.16$; HDL-C: $0.56 \pm 0.20 \mathrm{mmol} / \mathrm{l}$ ) and found that they were either homozygotes or compound heterozygotes for LOF mutations predicted to produce truncated ANGPTL3 proteins (p.G400Vfs*5, p.I19Lfs*22/p.N147*) (Table 2 \& Figure 1) [34]. Martín-Campos et al. resequenced the ANGPTL3 gene in three hypocholesterolemic individuals (TC: $1.65 \pm 0.36$; LDL-C: $1.12 \pm 0.27$; HDL-C: $0.39 \pm 0.07$; TG: $0.30 \pm 0.05 \mathrm{mmol} / \mathrm{l}$ ) belonging 


\begin{tabular}{|c|c|c|c|c|c|c|c|c|c|c|}
\hline \multirow[t]{2}{*}{ Exon } & \multirow[t]{2}{*}{ cDNA } & \multicolumn{2}{|c|}{ Protein } & \multicolumn{2}{|c|}{ PolyPhen-2 ${ }^{\dagger}$} & \multicolumn{2}{|c|}{ SIFT $^{\ddagger}$} & \multirow{2}{*}{$\begin{array}{l}\text { Mutation } \\
\text { testing }\end{array}$} & \multirow[t]{2}{*}{ Remark } & \multirow[t]{2}{*}{ Ref. } \\
\hline & & $\begin{array}{l}\text { Pro- } \\
\text { protein }\end{array}$ & Mature & $\begin{array}{l}\text { Hum div } \\
\text { damaging }\end{array}$ & $\begin{array}{l}\text { Hum var } \\
\text { damaging }\end{array}$ & $\begin{array}{l}\text { Human } \\
\text { protein }\end{array}$ & BLink & & & \\
\hline 1 & c. $121 \mathrm{G}>\mathrm{A}$ & p.D41N & D22N & Possibly & Possibly & Tolerated & Damaging & Disease-causing & HIS, high TG & [30] \\
\hline 1 & c. $167 \mathrm{G}>\mathrm{T}$ & p.G56V & G37V & Probably & Probably & Tolerated & Damaging & Disease-causing & $\begin{array}{l}\text { IT, low LDL-C } \\
\text { and HDL-C }\end{array}$ & [37] \\
\hline 1 & C.188 A>C & p.K63T & $\mathrm{K} 44 \mathrm{~T}$ & Probably & Probably & Tolerated & Damaging & Disease-causing & $\begin{array}{l}\text { AA, defective } \\
\text { LPL inhibition }\end{array}$ & [30] \\
\hline 1 & C. 214 T>C & p.F72L & F53L & Possibly & Benign & Tolerated & Tolerated & Disease-causing & HIS, high TG & [30] \\
\hline 1 & c. $272 A>G$ & p.E91G & E72G & Probably & Possibly & Tolerated & Tolerated & Disease-causing & $\begin{array}{l}\text { AA, defective } \\
\text { LPL inhibition }\end{array}$ & [30] \\
\hline 1 & c. $292 \mathrm{G}>\mathrm{A}$ & p.E98K & E79K & Benign & Benign & Tolerated & Tolerated & Disease-causing & HIS, high TG & [30] \\
\hline 1 & C. $392 \mathrm{~A}>\mathrm{C}$ & p.K131T & $\mathrm{K} 112 \mathrm{~T}$ & Probably & Probably & Tolerated & Tolerated & Disease-causing & EA, high TG & [30] \\
\hline 1 & c. 490 C>T & p.L164F & L145F & Possibly & Benign & Tolerated & Tolerated & Disease-causing & $\begin{array}{l}\text { AA, defective } \\
\text { LPL inhibition }\end{array}$ & [30] \\
\hline 2 & C. $518 A>G$ & p.N173s & N154S & Benign & Benign & Tolerated & Tolerated & Nonpathogenic & $\begin{array}{l}\text { AA, defective } \\
\text { LPL inhibition }\end{array}$ & [30] \\
\hline 4 & c.776 T>C & p.M259T & M240T & Benign & Benign & Tolerated & Damaging & Nonpathogenic & AA, SNP & [30] \\
\hline 5 & c. $863 \mathrm{G}>\mathrm{A}$ & p.R288Q & R269Q & Probably & Probably & Damaging & Damaging & Disease-causing & $\begin{array}{l}\text { EA, ANGPTL3 } \\
\text { secretion } \downarrow\end{array}$ & [30] \\
\hline 5 & c. $874 \mathrm{~T}>\mathrm{C}$ & p.S292P & S273P & Probably & Possibly & Damaging & Damaging & Disease-causing & $\begin{array}{l}\text { AA, ANGPTL3 } \\
\text { secretion } \downarrow\end{array}$ & [30] \\
\hline 5 & c.883 T>C & p.F295L & $\mathrm{F} 276 \mathrm{~L}$ & Probably & Probably & Damaging & Damaging & Disease-causing & $\begin{array}{l}\text { IT, low LDL-C } \\
\text { and HDL-C }\end{array}$ & [37] \\
\hline 6 & c. $995 \mathrm{G}>\mathrm{A}$ & p.R332Q & R313Q & Probably & Probably & Tolerated & Tolerated & Disease-causing & $\begin{array}{l}\text { IT, low LDL-C } \\
\text { and HDL-C }\end{array}$ & [37] \\
\hline 6 & c. $1123 \mathrm{G}>\mathrm{A}$ & p.E375K & E356K & Possibly & Benign & Tolerated & Tolerated & Disease-causing & $\begin{array}{l}\text { EA, ANGPTL3 } \\
\text { secretion } \downarrow\end{array}$ & [30] \\
\hline 7 & C. $1250 A>G$ & p.Y417C & Y398C & Probably & Probably & Damaging & Damaging & Disease-causing & $\begin{array}{l}\text { AA, ANGTL3 } \\
\text { secretion } \downarrow\end{array}$ & [30] \\
\hline 7 & c.1283 G>T & p.R428M & R409M & Probably & Probably & Tolerated & Tolerated & Disease-causing & EA, high TG & [30] \\
\hline $\begin{array}{l}{ }^{\dagger} \text { Please } s \\
{ }^{*} \text { Please } 5 \\
\text { AA: Afri } \\
\text { nucleotic }\end{array}$ & $\begin{array}{l}\text { see [101] for more } \\
\text { see [102] for more } \\
\text { can-American; } E\end{array}$ & $\begin{array}{l}\text { e details. } \\
\text { e details. } \\
\text { A: European }\end{array}$ & merican & celo & a sure & . & . & 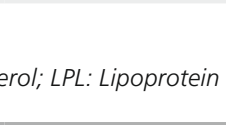 & MOCOP. Sin & \\
\hline
\end{tabular}

to two Spanish families with FHBL not linked to the $A P O B$ gene [35]. These individuals were found to be homozygotes for a 5-bp deletion resulting in a truncated ANGPTL3 protein (p.N121K*3) (Table 2 \& Figure I). More recently, Minicocci et al. resequenced the ANGPTL3 gene in a cluster of FHBL families identified in a small town in Central Italy and apparently not linked to $A P O B$ gene [36]. Overall, the ANGPTL3 gene was resequenced in nine index cases (plasma LDL-C $<$ fifth percentile), 28 first-degree relatives, 11 second-degree relatives and four spouses. This survey led to the identification of six homoygotes and 24 heterozygotes for a 2-bp change at nucleotides 50-51 of exon 1 (c.50 CC>GA), introducing a premature termination (p.S17*) (Table 2 \& Figure I) [36]. The plasma lipid profile found in the homoyzgotes (TC: $2.34 \pm 0.33$; LDL-C:
$1.24 \pm 0.40 ;$ HDL-C: $0.89 \pm 0.20 ;$ TG: $0.43 \pm 0.13 \mathrm{mmol} / \mathrm{l}$; ApoB: $0.48 \pm 0.10$; ApoA-I: $0.85 \pm 0.13 \mathrm{~g} / \mathrm{l}$ ) was consistent with a familial combined hypolipidemia phenotype, even though, on average, the values of TC, LDL-C and HDL-C were higher than those found in carriers of two LOF alleles belonging to the other kindreds investigated so far [33-35].

Finally, Noto et al. addressed the question of the impact of ANGPTL3 mutations as the cause of a combined hypolipidemia phenotype in subjects with the clinical diagnosis of putative FHBL [37]. From a large cohort of putative FHBL subjects (390 Italians and 523 Americans) they selected 78 individuals who presented with a combined hypolipidemia phenotype, defined by stringent criteria (i.e., $\mathrm{TC}<$ second percentile and HDL-C <second decile of the populations) based on the lipid profile of the four carriers of 
two mutant alleles in family ' $F$ ' [33]. The analysis of $A P O B$ and $A N G P T L 3$ genes revealed that none of these subjects had mutations in the $A P O B$ gene, while eight of them were carriers of the rare $A N G P T L 3$ mutation (four carriers of two mutant alleles and four carriers of one mutant allele). The mutations resulted in either truncated proteins or single rare nonconservative amino acid substitutions expected to be deleterious for ANGPTL3 function (Tables I \& $\mathbf{2}$ \& Figure I). In this study, the plasma lipid profile of carriers of two mutant $A N G P T L 3$ alleles (TC: $1.58 \pm 0.36$; LDL-C: $0.90 \pm 0.34$; HDL-C: $0.46 \pm 0.15$; TG: $0.45 \pm 0.28 \mathrm{mmol} / \mathrm{l}$ ) was fairly similar to that found in other subjects with familial combined hypolipidemia [33-35].

While these studies convincingly demonstrate that the presence of two LOF alleles of ANGPTL3 is associated with the combined hypolipidemia phenotype, the features of the lipid phenotype in carriers of one LOF allele are not yet clearly defined. In the original family ' $F$ ', reinvestigated by Musurunu et al. [33], the carriers of one mutant allele were found to have values of LDL-C and TG intermediate between those of the carriers of two mutant alleles and the noncarrier relatives. In sharp contrast, the levels of HDL-C were similar to those found in noncarriers, thus suggesting the concept that the low LDL-C and TG phenotype was transmitted as a codominant trait while the low HDL-C level was transmitted as a recessive trait [33]. However, at first sight, the results of the other studies do not support this contention [34-37]. The plasma LDL-C and TG levels in carriers of one mutant allele of the Italian kindreds or the Spanish kindreds were not significantly different from noncarriers $[34,35]$. On the other hand, Minicocci et al., who investigated a large number of carriers of the LOF allele $p . S 17^{*}$ found that these individuals had lower TC and HDL-C levels than noncarriers [36]. Finally, 12 heterozygous carriers of frameshift mutations (leading to premature truncation of the ANGPTL3 protein) found that the participants of the DHS trial had significantly lower plasma levels of LDL-C than noncarriers (median $2.00 \mathrm{mmol} / \mathrm{l}$ vs median $2.68 \mathrm{mmol} / \mathrm{l}$ in noncarriers; $p=0.03$ ). They also showed a reduction in plasma TG levels of borderline significance (median 0.81 vs $1.08 \mathrm{mmol} / \mathrm{l}$; $\mathrm{p}=0.08)$ [33].

To clarify this issue, the present authors tried to compare the plasma lipid profiles of the 94 heterozygous carriers of LOF mutations reported so far with those of 45 noncarrier family members [33-37]. This comparison showed that heterozygotes had significantly lower levels of TC, LDL-C and TG, but not significantly reduced levels of HDL-C compared with noncarrier family members (Table 3 \& Figure 4). Although more extensive studies are required, the result of this comparison would be consistent with a codominant transmission of the low LDL-C/TG trait and a recessive transmission for the low HDL-C trait.

\section{Table 2. ANGPTL3 nonsense and frameshift mutations.}

\begin{tabular}{|c|c|c|c|c|c|c|c|c|}
\hline Exon & CDNA $^{\dagger}$ & Pro-protein ${ }^{\dagger}$ & Previously reported as & Ethnic background & HO (n) & CHE (n) & HE (n) & Ref. \\
\hline 1 & c. $50-51$ CC > GA & p.S17* & - & EA, IT & 8 & 4 & 60 & {$[33,36,37]$} \\
\hline 1 & c.55delA & p. 119 Lfs $^{*} 22$ & - & IT & - & 3 & 3 & [34] \\
\hline 1 & c.247delT & p.Y83Mfs*17 & Y88fsX99 & AA & - & - & 1 & [33] \\
\hline 1 & c.286_288delGAA & p.E96del & c.283_284delGAA，E95del & IT & 1 & - & 1 & {$[36,37]$} \\
\hline 1 & c.361_365delAACTC & p.N121Kfs*3 & $\begin{array}{l}\text { c.355_360delAACTC, } \\
\text { E119fsX8 }\end{array}$ & IT & - & - & 1 & [37] \\
\hline 1 & c.362_363insCTCAT & p.K123Sfs*8 & c.362insCTCAT, N121fsX9 & EA & - & - & 1 & [37] \\
\hline 1 & c.363_364delCT & p.S122Kfs*3 & FsS122 & IT & - & - & 1 & [36] \\
\hline 1 & c.363_367delCTCAA & p.N121Kfs*3 & $\mathrm{N} 121 \mathrm{LX} 2$ & SP & 3 & - & 4 & [35] \\
\hline 1 & c.364_367delTCAA & p.S122Nfs*6 & $\mathrm{S} 122 \mathrm{fs} \times 128$ & $A A, E A$ & - & - & 2 & {$[30,33]$} \\
\hline 1 & c. $385 \mathrm{G}>\mathrm{T}$ & p.E129* & - & EA & - & 4 & 6 & {$[33,37]$} \\
\hline 1 & c.439_442delAACT & p.N147* & N147fsX148 & IT, AA, EA & 1 & 4 & 2 & {$[33,34,37]$} \\
\hline 2 & c.575delA & p.Q192Rfs*5 & Q192fsX196 & $\mathrm{AA}$ & - & - & 5 & {$[30,33]$} \\
\hline 5 & c.862_864delCGA & p.R288del & - & $A A$ & - & - & 1 & [30] \\
\hline 6 & c.1167delA & p.G390Dfs*24 & K389fsX413 & EA & - & - & 1 & [33] \\
\hline IVS6 & c. $1198+1 G>T$ & p.G400Vfs*5 & - & IT & 1 & - & 2 & {$[30,34]$} \\
\hline 7 & c.1333_1334insT & p.K445Ifs*16 & K445fsX459 & AA & - & - & 1 & {$[30,33]$} \\
\hline
\end{tabular}




\section{ANGPTL3 gene variants identified in humans}

Resequencing of the coding sequence of the ANGPTL3 gene in population samples and in subjects with combined hypolypidemia has led to the identification of 17 missense mutations,
15 mutations resulting in premature termination codons (nonsense and frameshift mutations) and one splice-site mutation (Tables I \& 2). Most of the missense mutations involved highly conserved amino acids and turned out to be possibly damaging in silico and eight of them were found

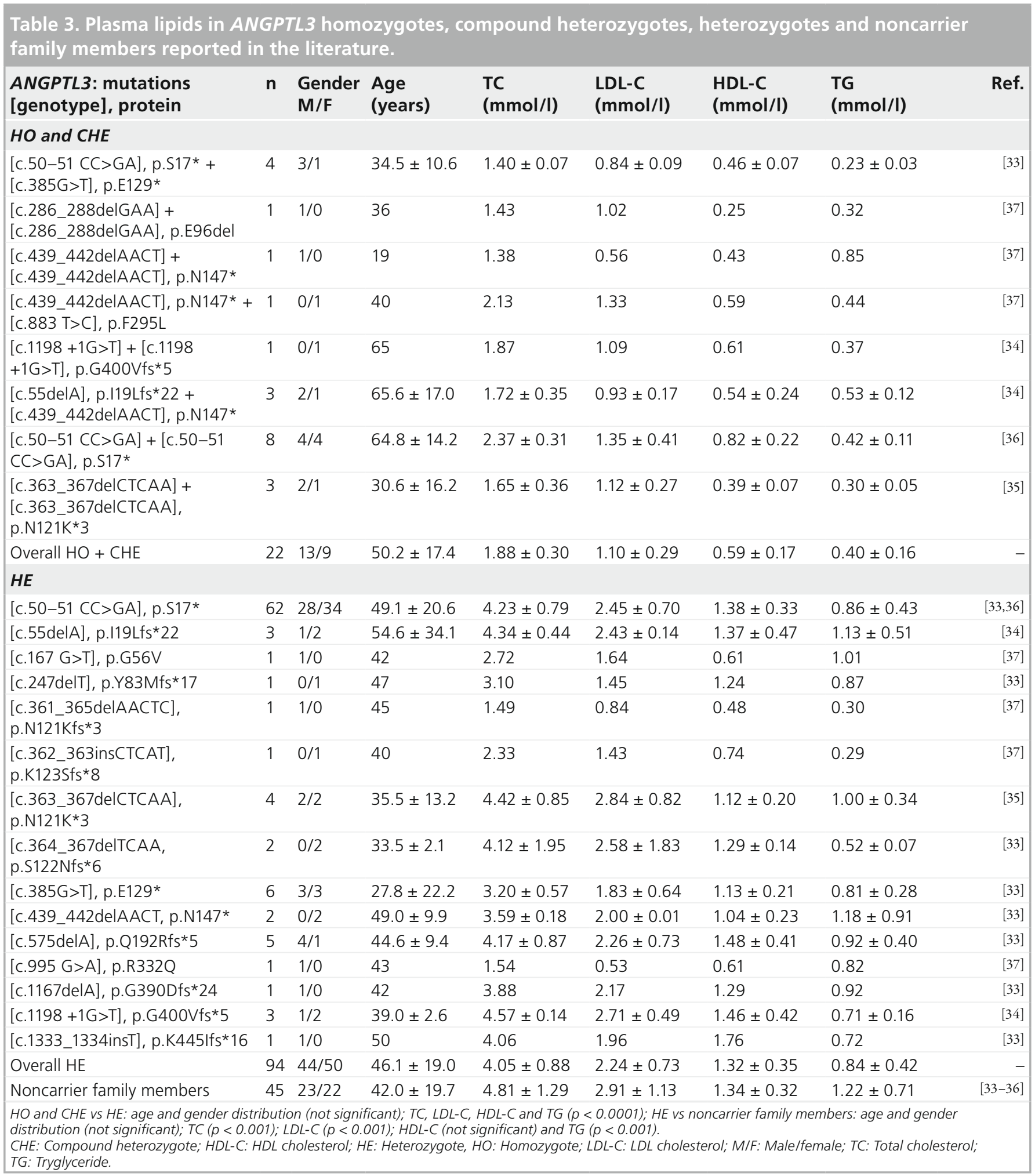


to either reduce the secretion of ANGPTL3 or to abolish its capacity to inhibit LPL in vitro [30]. The splice site mutation (c.1198+1G>T) in intron 6 was found to cause the partial retention of intron 6 in the mature mRNA [34].

\section{Plasma levels of ANGPTL3 in familial combined hypolipidemia}

The present authors' group found that in carriers of two LOF alleles, no ANGPTL3 protein was detectable in plasma, while in carriers of one LOF allele, the mean level of plasma ANGPTL3 was approximately $40 \%$ of that found in control subjects [34]. These findings were confirmed in the study of Minicocci et al., which showed that homozygotes for LOF alleles (p.S17*) had no detectable ANGPTL3 protein in their plasma, while in heterozygotes, the level of ANGPTL3 was reduced by $42 \%$ [36]. Overall, the carriers of two LOF alleles can be regarded as having a complete ANGPTL3 deficiency (ANGPTL3 $3^{-/}$), possibly as the result of defective secretion of the mutant truncated proteins or its rapid clearance from the plasma compartment. Such defective secretion may be due to accelerated intracellular degradation or defective synthesis of the mutant ANGPTL3 (due to the rapid degradation of the corresponding mRNA containing a premature termination codon).

\section{Plasma lipoproteins in familial combined hypolipidemia \\ - ApoB-containing lipoproteins}

ApoB turnover studies conducted on selected members of family ' $F$ ' indicated marked changes of ApoB turnover [33]. The VLDLApoB production rate was reduced by $70 \%$ in carriers of two mutant alleles $(n=2)$ and by $40 \%$ in carriers of one mutant allele $(n=3)$. The LDL-ApoB fractional catabolic rate was increased by approximately $50 \%$ in carriers of two mutant alleles and approximately $40 \%$ in carriers of one mutant allele. These findings are consistent with the gene-dosage effect for VLDL production and LDL fractional catabolic rates, and provide an explanation for the codominant transmission of the low LDL-C and TG trait found in heterozygotes of family ' $F$ '. The link between these changes in the metabolism of ApoB-containing lipoproteins and ANGPTL3 deficiency is not clearly understood. The decreased VLDL-ApoB production rate would imply some functional role of ANGPTL3 in the VLDL secretion pathway (including ApoB lipidation, intracellular assembly of VLDL

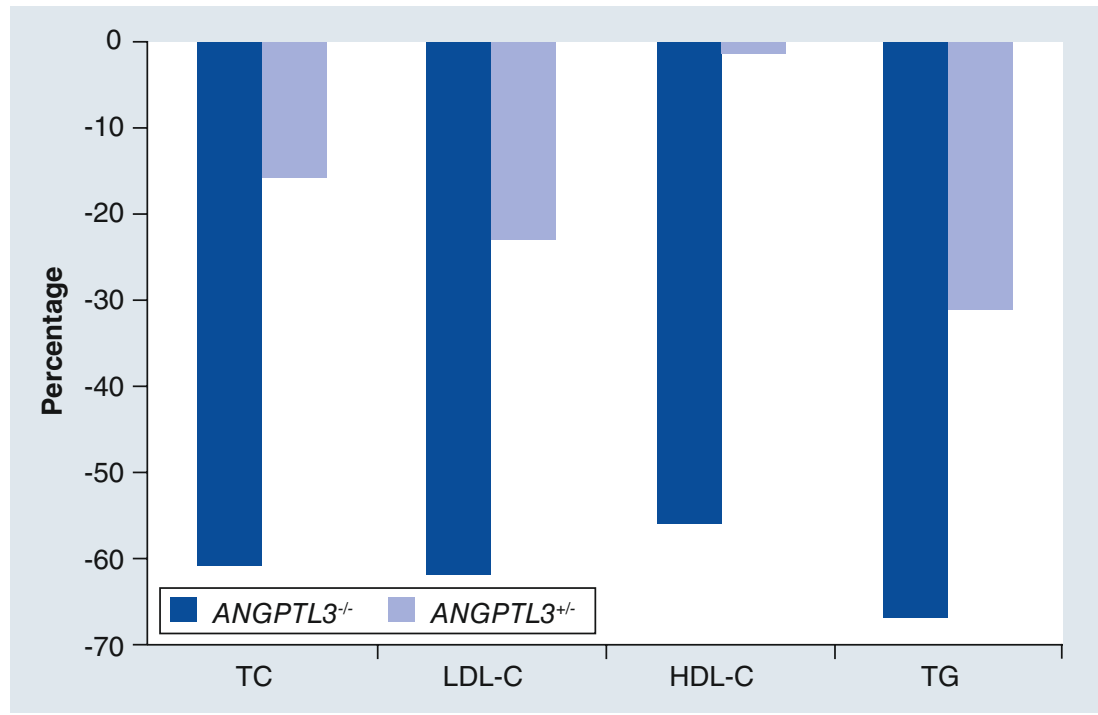

Figure 4. Percentage reduction of plasma lipids in carriers of loss-offunction mutations in ANGPTL3. Mean percentage reduction of plasma lipids in carriers of one $(n=22)$ or two $(n=94)$ mutant alleles of the ANGPTL3 gene with respect to the lipid levels of noncarrier family members $(n=45)$. The mutant alleles are listed in Table 3

HDL-C: HDL cholesterol; LDL-C: LDL cholesterol; TC: Total cholesterol; TG: Triglyceride.

particles and transfer of these particles from the lumen of the endoplasmic reticulum to the Golgi, among others). Under physiological conditions, ANGPTL3 might serve as a chaperone molecule functioning to protect ApoB from intracellular degradation, thus facilitating the assembly of a higher number of VLDL particles. In ANGPTL3 deficiency, this 'protective' effect on ApoB would be lost and fewer VLDL particles would be secreted. This appears to be an attractive hypothesis, even though the studies on hepatic TG secretion in ANGPTL3 deficient mice do not appear to support this contention. In KK/Snk hypolipidemic mice, the in vivo secretion rate of plasma TG showed the tendency to be lower (by 15\%) compared with the wild-type hypertriglyceridemic KK mice, but the difference was not significant [7]. A similar observation was made in ANGPTL3-null mice [10]. However, in vivo hepatic TG secretion in $\mathrm{KK} / \mathrm{Snk}$ mice was found to be significantly reduced (by 25\%) compared with that of normotriglyceridemic control mice [38].

VLDL turnover studies conducted in hypolipidemic KK/Snk mice revealed that these mice exhibited enhanced VLDL TG clearance compared with KK mice [7]. Similar turnover studies conducted in these mice on the ApoE knockout (KO) background showed that ${ }^{3} \mathrm{H}$-labeled TGs and ${ }^{125} \mathrm{I}$-labeled VLDL ApoB were removed more rapidly from the circulation in 
the hypolipidemic KK/Snk ApoE KO compared with ApoE KO mice [38]. In addition, the oral fat-load test showed that in KK/Snk mice, the postprandial plasma TG increase was much lower than that found in control mice in the presence, as well as in the absence, of ApoE, a finding consistent with increased activity of LPL and hepatic lipase [38]. In view of these observations, one would expect an increased VLDL fractional catabolic rate and a faster postprandial removal of TG-rich lipoproteins to be present in humans with complete ANGPTL3 deficiency.

The increased LDL-ApoB catabolic rate might be the result of unrestrained EL activity (due to the loss of the inhibitory effect of ANGPTL3). The hepatic overexpression of EL in mouse models with elevated levels of ApoBcontaining lipoproteins (ApoE deficient, LDL receptor deficient and human $A p o B$ transgenic mice) resulted in markedly reduced VLDL/LDL cholesterol, phospholipids and ApoB levels, accompanied by a significant increase in LDL apolipoprotein and phospolipid catabolism [39].

- HDLs

Complete ANGPTL3 deficiency (ANGPTL3 ${ }^{-/-}$) in humans was found to be associated not only with a striking reduction of plasma HDL-C and ApoA-I levels, but also with changes in HDL subclass distribution consisting of a striking reduction of LpAI and LpAI/AII particles (-70 and $-30 \%$ respectively), a decreased level of pre- $\beta-H D L$ and a reduced HDL size. In addition, the lecithin:cholesterolacyltransferase activity and the cholesterol esterification rate were found to be reduced or close to the lower limits of the normal range [34].

Some of the changes in HDL found in ANGPTL3 ${ }^{-1-}$ subjects are probably due to the increased EL activity, as suggested by animal and human studies. Overexpression of EL in mice resulted in reduced plasma HDL levels and, conversely, EL KO mice showed significant increases in HDL levels [20]. In addition, EL overexpression in mice induced a dose-dependent increase in the fractional catabolic rate of HDLapolipoprotein and in renal ApoA-I catabolism [40], and produced an enhanced hepatic uptake of HDL-C through the SR-BI [41]. In humans, a negative correlation was found between HDL-C concentrations and both pre- and post-heparin EL concentrations [42]. Furthermore, nuclear magnetic resonance analysis of plasma HDL revealed a negative association between postheparin EL concentrations and large HDL particles, and a positive association of EL mass with small HDL particles [42].

Further support for the role of increased EL activity on the HDL changes found in ANGPTL $3^{-1-}$ subjects emerged from the resequencing of the LIPG gene (the gene encoding EL) in individuals with extreme levels of plasma HDL-C. Minor alleles of two variants (rs4299883 and rs2156552) of the $L I P G$ gene reported to be highly associated with decreased HDL-C in the Global Lipid Genetics Consortium GWAS were tested for the association with HDL-C and HDL subtypes in subjects of the Framigham Heart Study [29]. The minor alleles of these variants were found to be associated with decreased $\mathrm{HDL}-\mathrm{C}, \mathrm{HDL}_{2}, \mathrm{HDL}_{3}$, HDL particle size and ApoA-I level. In keeping with these findings, these minor alleles were also associated with increased plasma EL [43].

The quantitative and qualitative changes in HDL-C observed in subjects with complete ANGPTL3 deficiency (ANGPTL3 ${ }^{--1}$ ) were also associated with a reduced capacity of their sera (depleted of ApoB-containing lipoproteins) to act as acceptors of cholesterol effluxed from cells through various pathways (ABCA1-, SR-BI- and ABCG1-mediated pathways) [34]. This suggests that reverse cholesterol transport might be impaired in these individuals. In subjects with partial ANGPTL3 deficiency $\left(A N G P T L 3^{+/}\right)$, only the level of pre- $\beta$-HDL was found to be reduced.

Consistent with this finding, the ApoBdepleted sera of these subjects had a reduced capacity to act as acceptors of cholesterol effluxed via the ABCA1-mediated pathway.

\section{Familial combined hypolipidemia $\&$ atherosclerosis}

Given the striking reduction of plasma HDL-C and ApoA-I, and the reduced capacity of the ApoB-depleted sera (a parameter found to show an inverse relationship with carotid intimamedia thickness and to be a strong predictor of coronary heart disease) [44], one would expect evident manifestations of preclinical/clinical atherosclerosis to be present in ANGPTL3 ${ }^{-1-}$ subjects. On the other hand, these individuals have had a strikingly reduced plasma level of ApoB-containing lipoproteins (VLDL and LDL) since birth, a condition that might have counterbalanced any proatherogenic effect derived from low HDL and reduced HDL-mediated cholesterol efflux capacity.

More specifically, no clinical overt manifestations of atherosclerotic cardiovascular disease 
was reported in $A N G P T L 3^{-1-}$ subjects of family ' $\mathrm{F}$ ' ( $\mathrm{n}=4$ adult subjects aged from 23 to 44 years) [33], of the Italian kindreds $(\mathrm{n}=4$ adult subjects aged from 53 to 85 years) [34] and of the Spanish kindreds ( $\mathrm{n}=2$ adult subjects aged 39 and 41 years) [35]. In the large survey by Minicocci et al., carriers of two mutant $A N G P T L 3$ alleles ( $\mathrm{n}=8$ homozygotes with a mean age of $64.8 \pm 14.2$ years) did not show an increased risk for ischemic heart disease, compared with noncarriers of comparable age living in the same geographical district [36]. Therefore, the data available so far does not point to an increased risk of atherosclerotic cardiovascular disease in $A N G P T L 3^{-1-}$ subjects with combined hypolipidemia. The lack of a relationship between low HDL-C/reduced cholesterol efflux capacity of sera (as previously mentioned) and an increased susceptibility to atherosclerosis is probably due to the markedly reduced level of ApoB-containing lipoproteins found in these individuals. It is likely that the lifelong exposure to low levels of VLDL and LDL might counterbalance the putative 'proatherogenic' effect related to low HDL-C. It seems interesting to consider these findings in the light of the observations made in the a KK/Snk/ApoE KO mice. Besides an extremely low level of HDL (associated with the absence of ApoE), these mice exhibited significant reductions in VLDL-TG, VLDL cholesterol and plasma ApoB compared with the ApoE KO mice. These mice developed threefold smaller atherogenic lesions in the aortic sinus compared with the control ApoE KO mice [38].

\section{Familial combined hypolipidemia \& liver disease}

It is well established that in $A P O B$-linked FHBL, severe fatty liver disease is present with a prevalence rate of approximately $20-30 \%$ [32]. In this condition, fatty liver is caused by an impaired secretion of ApoB-containing lipoproteins that leads to an accumulation of lipids (mainly TG) in the hepatocytes. Fatty liver has not been reported so far in subjects with ANGPTL3 ${ }^{-/}$ [33-35,37] and no increase in the prevalence of chronic liver disease was reported in carriers of two mutant ANGPTL3 alleles in the survey by Minicocci et al. [36].

\section{Familial combined hypolipidemia \& hematopoiesis}

Studies in mice have suggested that ANGPTL3 is involved in the regulation of hematopoietic stem cell (HSC) activity. The addition of ANGPTL3 to a culture of mouse bone marrow side population of $\mathrm{CD} 45^{+} \mathrm{Sca}-1^{+}$cells, together with saturating levels of other growth factors, resulted in a marked expansion of long-term mouse HSC number [45]. In line with this observation, HSCs were found to be decreased in number in Angptl3 ${ }^{-1-}$ mice and wild-type HSCs transplanted into Angptl3 ${ }^{-1-}$ mice exhibited impaired repopulation [46]. In Angptl3 $3^{-/-}$mice, the lack of production of ANGPTL3 by endothelial and stromal cells of the bone marrow significantly decreased the ability to support expansion of repopulating HSCs. In addition, the bone marrow of $A n g p t 3^{-1-}$ was found to have a decreased number of granulocytes/monocytes progenitors (colonyforming unit granulocytes/monocytes) and B lymphoid progenitors (colony-forming unit B progenitor cells) compared with bone marrow of wild-type mice [46]. Although there are no reports of the presence of hematological disorders in humans with complete ANGPTL3 deficiency, it would be interesting to know whether these subjects have subtle changes in blood cell number and whether they are more susceptible to developing some kind of hematological disorders during life.

\section{Hypolipidemia in carriers of ANGPTL8 gene variants}

The observation in mice that ANGPTL8 regulates TG and fatty acid metabolism by controlling the activation of ANGPTL3 [21] raises the possibility that in humans, LOF mutations of ANGPTL8 are the cause of a combined hypolipidemia phenotype similar to that found in homozygous/compound heterozygous carriers of LOF mutations of ANGPTL3. Quagliarini et al. investigated the effect of a common NS sequence variant of ANGPTL8 (c.194C>T, rs2278426 resulting in tryptophan for arginine substitution, Arg59Trp or R59W) on plasma lipoproteins among the participants of the DHS trial [21]. This variant was found to be more common among Hispanics $(\mathrm{MAF}=26 \%)$ and African-Americans $(\mathrm{MAF}=18 \%)$ than European-Americans $(\mathrm{MAF}=5 \%)$. In African-Americans and Hispanics, Arg59Trp was associated with reduced LDL-C and HDL-C levels without changes in TG levels. This association was replicated in African-American participants in the ARIC study. In the European-Americans of ARIC, the Arg59Trp variant was associated with significantly lower levels of HDL-C, but not LDL-C. Taken together, these observations indicate that putative LOF variants of ANGPTL8 may induce a hypolipidemia phenotype, which 
appears to be different from that observed in carriers of LOF mutations of ANGPTL3. Only the identification of individuals carrying two null ANGPTL8 alleles (ANGPTL $8^{-1}{ }^{-}$individuals) will clarify this issue.

\section{Conclusion}

Almost 10 years have elapsed since the report of a severe recessive form of hypotriglyceridemia in a mouse strain carrying a LOF mutation of the ANGPTL3 gene, expected to lead to a complete ANGPTL3 deficiency. A severe hypolipidemia characterized by reduced levels of both TG and TC was subsequently found in ANGPTL3 $\mathrm{KO}$ mice. In vivo and in vitro studies have demonstrated that ANGPTL3 acts as an inhibitor of both LPL and EL. GWAS in humans indicated an association of ANGPTL3 locus variants with plasma $\mathrm{TG}$. The resequencing of the ANGPTL3 gene in populations demonstrated an enrichment of LOF mutations in individuals in the lowest quartile of plasma TGs. Recently, the genomic investigation of a large family with FHBL, not linked to the $A P O B$ or PCSK9 gene, led to the identification of LOF mutations in the ANGPTL3 gene as the cause of

\section{Executive summary}

\section{ANGPTL3 \& lipoprotein metabolism in mice \& humans}

- Early studies in mice had demonstrated that loss-of-function mutations of ANGPTL3 were associated with a severe hypolipidemia affecting all plasma lipoprotein classes, specifically the triglyceride-rich lipoproteins. ANGPTL3 was found to inhibit lipoprotein lipase and endothelial lipase.

\section{Activation of ANGPTL3 by ANGPTL8, a new member of ANGPTL family}

- ANGPTL8 (also called lipasin) is a new member of the angiopoietin-like protein family found in human and mouse plasma. ANGPTL8 activates ANGPTL3 by facilitating its proteolytic cleavage and the release of the $\mathrm{N}$-terminal domain, which is responsible for the inhibition of lipoprotein lipase and endothelial lipase.

\section{Plasma level of ANGPTL3 \& correlation with plasma lipids}

- In mice and humans, the plasma level of ANGPTL3 appears to be directly correlated to that of plasma HDL cholesterol (HDL-C).

\section{ANGPTL3 gene variants \& plasma lipids in population studies}

- Genome-wide association studies have documented an association of single nucleotide polymorphisms located close to the ANGPTL3 locus and a reduction of plasma triglyceride. Rare loss-of-function mutations of the ANGPTL3 gene were found in individuals with low plasma triglycerides in population studies.

\section{ANGPTL3 \& familial combined hypolipidemia}

" Loss-of-function mutations in the ANGPTL3 gene in the homozygous/compound heterozygous state were found to be the cause of a marked reduction of all plasma lipoprotein classes, a condition designated familial combined hypolipidemia. This disorder does not seem to be associated with tangible clinical manifestations. Heterozygous carriers of these mutations appear to have reduced plasma LDL cholesterol and triglyceride levels.

\section{Plasma level of ANGPTL3 in familial combined hypolipidemia}

" ANGPTL3 protein was not detectable in plasma of subjects with familial combined hypolipidemia, indicating that the presence of two loss-of-function alleles leads to a complete ANGPTL3 deficiency.

\section{Plasma lipoproteins in familial combined hypolipidemia}

- In vivo kinetic studies showed a marked reduction of VLDL-ApoB production rate and an increased LDL-ApoB catabolic rate in carriers of two mutant ANGPTL3 alleles. The subjects have marked changes in plasma HDL subclasses characterized specifically by a reduction of HDL size and of the pre- $\beta$ HDL particles.

- ApoB-depleted sera of these subjects have a reduced capacity to act as acceptors of cholesterol released from the cells in vitro through the various efflux pathways. This suggests a defect in the early steps of reverse cholesterol transport.

\section{Familial combined hypolipidemia \& atherosclerosis}

" Despite the striking reduction of plasma HDL-C and ApoA-I, no premature atherosclerosis has been reported so far in individuals with familial combined hypolipidemia.

\section{Familial combined hypolipidemia \& liver disease}

- Despite a reduction of plasma ApoB-containing lipoproteins similar to that observed in familial hypobetalipoproteinemia, fatty liver disease has not been reported so far in subjects with familial combined hypolipidemia.

\section{Familial combined hypolipidemia \& hematopoiesis}

- In the bone marrow of ANGPTL3\% mice, the number of hematopoietic stem cells and that of granulocyte/monocyte progenitors, and B-lymphoid progenitors, was found to be reduced. A careful monitoring of the blood cell count is recommended in subjects with familial combined hypolipidemia.

\section{Hypolipidemia in carriers of ANGPTL8 gene variants}

- Sequencing of the ANGPTL8 gene in populations has led to the discovery of a common sequence variant (Arg59Trp) that was found to be associated, in the heterozygous state, with lower plasma LDL cholesterol and HDL-C levels. 
a severe hypolipidemia involving all lipoprotein classes (VLDL, LDL and HDL). This study prompted the resequencing of the ANGPTL3 gene in individuals with unexplained FHBL, showing a combined hypolipidemia phenotype. These surveys led to the identification of several individuals homozygous/compound heterozygous for LOF mutations causing complete ANGPTL3 deficiency. Familial combined hypolipidemia is transmitted as a recessive disorder. However, preliminary evidence indicates that heterozygotes may have reduced plasma LDL-C and TG, suggesting that this trait has a codominant transmission. In vivo kinetic studies indicated that individuals carrying two mutant ANGPTL3 alleles have a reduced VLDL-ApoB production rate and an increased LDL-ApoB catabolic rate. On the other hand, these individuals also have significant changes in HDL subpopulations and a reduced capacity of their sera to act as acceptors of cholesterol efflux from the cells in vitro, suggesting an impaired reverse cholesterol transport. Familial combined hypolipidemia does not seem to be associated with overt clinical manifestations. Recent studies have indicated that ANGPTL3 is activated by ANGPTL8, a novel member of the angiopoietin-like protein family. In some populations, a common missense variant of ANGPTL8 was found to be associated with reduced plasma levels of some lipoprotein classes.

\section{Future perspective}

The recent finding that complete ANGPTL3 deficiency is the cause of combined familial hypolipidemia raises several questions concerning the biochemical mechanism underlying this lipid disorder, the possible long-term effect of this hypolipidemia and the role of ANGPTL3 as a possible target for hypolipidemic drugs.

With regard to the mechanism, it would be interesting to know whether the role of ANGPTL3 is confined to the inhibition of LPL and EL, or also involves the assembly and secretion of ApoB-containing lipoproteins in the liver. It is possible that ANGPTL3 acts as a chaperone molecule that protects ApoB from intracellular

\section{References}

Papers of special note have been highlighted as:

= of interest

" - of considerable interest

1 Hato T, Tabata M, Oike Y. The role of angiopoietin-like proteins in angiogenesis and metabolism. Trends Cardiovasc. Med. 18(1), 6-14 (2008).

degradation before its incorporation into nascent lipoprotein particles. Another key issue is whether the unrestrained activity of EL expected to occur in complete ANGPTL3 deficiency has a negative effect on the functionality of HDL with regard to the protection against atherosclerosis.

The identification of individuals with complete ANGPTL3 deficiency will set the stage for a number of clinical investigations aimed at defining whether the combined hypolipidemia predisposes to complications, such as premature atherosclerosis (in view of the reduced level and the structural changes of HDL), fatty liver diseases (in view of the possible defect in VLDL production), defects in steroid hormone production (in view of the reduced availability of the LDL- and HDL-derived cholesterol in the steroidogenic tissues) or hematological disorders (in view of the stimulatory effect of ANGPTL3 on hematopoietic stem cells in the bone marrow).

Finally, the observation that individuals heterozygous for LOF mutations with partial ANGPTL3 deficiency have reduced levels of LDL-C and TG may prompt the search for therapeutic strategies aimed at reducing the inhibitory effect of ANGPTL3 on LPL. Partial inhibition of ANGPTL3 is expected to be associated with an increased catabolism of TG-rich lipoproteins.

\section{Financial \& competing interests disclosure}

This study was supported by a grant from the Department of Health of the Emilia-Romagna Region (Dialer Project) (S Calandra), grants from the Fondazione Cassa di Risparmio di Modena, FCRM (P Tarugi), the University of Genova (S Bertolini) and the University of Palermo (M Averna). The authors thank Stefano Romeo for his personal input, which led to a more accurate description of some mutations of the ANGPTL3 gene found in the Dallas

Heart Study. The authors have no other relevant affliations or financial involvement with any organization or entity with a financial interest in or financial conflict with the subject matter or materials discussed in the manuscript apart from those disclosed.

No writing assistance was utilized in the production of this manuscript.

2

Miida T, Hirayama S. Impacts of angiopoietinlike proteins on lipoprotein metabolism and cardiovascular events. Curr. Opin. Lipidol. 21(1), 70-75 (2010).

3 Mattijssen F, Kersten S. Regulation of triglyceride metabolism by angiopoietin-like proteins. Biochim. Biophys. Acta 1821(5), 782-789 (2012)
4 Bauer RC, Stylianou IM, Rader DJ. Functional validation of new pathways in lipoprotein metabolism identified by human genetics. Curr. Opin. Lipidol. 22(2), 123-128 (2011).

5 Lichtenstein L, Kersten S. Modulation of plasma TG lipolysis by angiopoietin-like proteins and GPIHBP1. Biochim. Biophys. Acta 1801(4), 415-420 (2010). 
6 Koishi R, Ando Y, Ono M et al. Angptl3 regulates lipid metabolism in mice. Nat. Genet. 30(2), 151-157 (2002)

7 Shimizugawa T, Ono M, Shimamura M et al. ANGPTL3 decreases very low density lipoprotein triglyceride clearance by inhibition of lipoprotein lipase. J. Biol. Chem. 277(37), 33742-33748 (2002).

8 Ono M, Shimizugawa T, Shimamura M, Yoshida K et al. Protein region important for regulation of lipid metabolism in angiopoietinlike 3 (ANGPTL3): ANGPTL3 is cleaved and activated in vivo. J. Biol. Chem. 278(43), 41804-41809 (2003).

9 Köster A, Chao YB, Mosior M et al. Transgenic angiopoietin-like (angptl)4 overexpression and targeted disruption of angptl4 and angptl3: regulation of triglyceride metabolism. Endocrinology 146(11), 4943-4950 (2005).

10 Fujimoto K, Koishi R, Shimizugawa T, Ando Y. Angptl3-null mice show low plasma lipid concentrations by enhanced lipoprotein lipase activity. Exp. Anim. 55(1), 27-34 (2006).

11 Lee EC, Desai U, Gololobov G et al. Identification of a new functional domain in angiopoietin-like 3 (ANGPTL3) and angiopoietin-like 4 (ANGPTL4) involved in binding and inhibition of lipoprotein lipase (LPL). J. Biol. Chem. 284(20), 13735-13745 (2009).

- Identification of the specific domain of ANGPTL3 involved in the interaction with lipoprotein lipase.

12 Shan L, Yu XC, Liu Z et al. The angiopoietinlike proteins ANGPTL3 and ANGPTL4 inhibit lipoprotein lipase activity through distinct mechanisms. J. Biol. Chem. 284(3), 1419-1424 (2009).

II Demonstration that ANGPTL3 reduces lipoprotein lipase catalytic activity, but does not alter its self-inactivation rate.

13 Liu J, Afroza H, Rader DJ, Jin W. Angiopoietin-like protein 3 inhibits lipoprotein lipase activity through enhancing its cleavage by proprotein convertases. J. Biol. Chem. 285(36), 27561-27570 (2010).

- Demonstration that, in the presence of cells, ANGPTL3 enhances the cleavage of lipoprotein lipase by pro-protein convertases.

14 Adeyo O, Goulborne CN, Bensadoun A, Beigneux AP, Fong LG, Young SG. Glycosylphosphatidylinositol-anchored highdensity lipoprotein-binding protein 1 and the intravascular processing of triglyceride-rich lipoproteins. J. Intern. Med. 272(6), 528-540 (2012).

15 Nilsson SK, Anderson F, Ericsson $\mathrm{M}$ et al. Triacylglycerol-rich lipoproteins protect lipoprotein lipase from inactivation by ANGPTL3 and ANGPTL4. Biochim. Biophys. Acta 1821(10), 1370-1378 (2012).

16 Sonnenburg WK, Yu D, Lee EC et al. GPIHBP1 stabilizes lipoprotein lipase and prevents its inhibition by angiopoietin-like 3 and angiopoietin-like 4. J. Lipid Res. 50 (12), 2421-2429, (2009).

17 Shimamura M, Matsuda M, Yasumo H et al. Angiopoietin-like protein3 regulates plasma HDL cholesterol through suppression of endothelial lipase. Arterioscler. Thromb. Vasc. Biol. 27(2), 366-372 (2007).

18 Jin W, Wang X, Millar JS et al. Hepatic proprotein convertases modulate $\mathrm{HDL}$ metabolism. Cell Metab. 6(2), 129-136 (2007).

19 Jin W, Fuki IV, Seidah NG, Benjannet S, Glick JM, Rader DJ. Proprotein convertases are responsible for proteolysis and inactivation of endothelial lipase. J. Biol. Chem. 280 (44), 36551-36559 (2005).

20 Jaye M, Lynch KJ, Krawiec J et al. A novel endothelial-derived lipase that modulates HDL metabolism. Nat. Genet. 21(4), 424-428 (1999)

21 Quagliarini F, Wang Y, Kozlitina J et al. Atypical angiopoietin-like protein that regulates ANGPTL3. Proc. Natl Acad. Sci. USA 109(48), 19751-19756 (2012).

- Demonstration that ANGPTL3 is activated by ANGPTL8, a novel member of the family of angiopoietin-like proteins.

22 Ren G, Kim JY, Smas CM. Identification of RIFL, a novel adipocyte-enriched insulin target gene with a role in lipid metabolism. Am. J. Physiol. Endocrinol. Metab. 303(3), e334-e351 (2012)

23 Zhang R. Lipasin, a novel nutritionallyregulated liver-enriched factor that regulates serum triglyceride levels. Biochem. Biophys. Res. Commun. 424(4), 786-792 (2012).

24 Tang T, Li L, Tang J et al. A mouse knockout library for secreted and transmembrane proteins. Nat. Biotechnol. 28(7), 749-755, (2010).

25 Hatsuda S, Shoji T, Shinohara K et al. Association between plasma angiopoietin-like protein 3 and arterial wall thickness in healthy subjects. J. Vasc. Res. 44(1), 61-66 (2007).

26 Robciuc MR, Tahvanainen E, Jauhiainen M, Ehnholm C. Quantitation of serum angiopoietin-like proteins 3 and 4 in a Finnish population sample. J. Lipid Res. 51(4), 824-831 (2010)

27 Moon HD, Nakajima K, Kamiyama K, Takanashi K, Sakurabayashi I, Nagamine T. Higher frequency of abnormal serum angiopoietin-like protein 3 than abnormal cholesteryl ester transfer protein in Japanese hyperalphalipoproteinemic subjects. Clin. Chim. Acta 398(1-2), 99-104 (2008).

28 Kathiresan S, Melander O, Guiducci C et al. Six new loci associated with blood low-density lipoprotein cholesterol, high-density lipoprotein cholesterol or triglycerides in humans. Nat. Genet. 40(2), 189-197 (2008).

29 Teslovich TM, Musunuru K, Smith AV et al. Biological, clinical and population relevance of 95 loci for blood lipids. Nature 466(7307), 707-713 (2010)

30 Romeo S, Yin W, Kozlitina J, Pennacchio LA et al. Rare loss-of-function mutations in ANGPTL family members contribute to plasma triglyceride levels in humans. J. Clin. Invest. 119(1), 70-79 (2009).

31 Pulai JI, Neuman RJ, Groenewegen AW, Wu J, Schonfeld G. Genetic heterogeneity in familial hypobetalipoproteinemia: linkage and nonlinkage to the apoB gene in Caucasian families. Am. J. Med. Genet. 76(1), 79-86 (1998).

32 Tarugi P, Averna M. Hypobetalipoproteinemia: genetics, biochemistry, and clinical spectrum. Adv. Clin. Chem. 54, 81-107 (2011).

33 Musunuru K, Pirruccello JP, Do R et al. Exome sequencing, ANGPTL3 mutations, and familial combined hypolipidemia. N. Engl. J. Med. 363(23), 2220-2227 (2010).

- First identification of mutations in the ANGPTL3 gene as the cause of familial combined hypolipidemia.

34 Pisciotta L, Favari E, Magnolo L et al. Characterization of three kindreds with familial combined hypolipidemia caused by loss-of-function mutations of ANGPTL3.Circ. Cardiovasc. Genet. 5(1), 42-50 (2012)

- Familial combined hypolipidemia is associated with changes in the subtypes and functionality of HDL.

35 Martín-Campos JM, Roig R, Mayoral C et al. Identification of a novel mutation in the ANGPTL3 gene in two families diagnosed of familial hypobetalipoproteinemia without APOB mutation. Clin. Chim. Acta 413(5-6), 552-555 (2012).

36 Minicocci I, Montali A, Robciuc MR et al. Mutations in the ANGPTL3 gene and familial combined hypolipidemia: a clinical and biochemical characterization. J. Clin. Endocrinol. Metab. 97(7), e1266-e1275 (2012).

- Identification of a cluster of families with familial combined hypolipidemia sharing the same $A N G P T L 3$ gene mutation.

37 Noto D, Cefalù AB, Valenti V et al. Prevalence of $A N G P T L 3$ and $A P O B$ gene mutations in subjects with combined hypolipidemia. Arterioscler. Thromb. Vasc. Biol. 32(3), 805-809 (2012). 


\section{Familial combined hypolipidemia due to mutations in the ANGPTL3 gene $\mid$ REVIEW}

- Loss-of-function mutations of ANGPTL3 were found in a subset of subjects with the clinical diagnosis of familial hypobetalipoproteinemia associated with low HDL cholesterol levels.

38 Ando Y, Shimizugawa T, Takeshita $\mathrm{S}$ et al. A decreased expression of angiopoietin-like 3 is protective against atherosclerosis in apoE-deficient mice. J. Lipid Res. 44(6), 1216-1223 (2003).

39 Broedl UC, Maugeais C, Millar JS et al. Endothelial lipase promotes the catabolism of apoB-containing lipoproteins. Circ. Res. 94(12), 1554-1561 (2004).

40 Maugeais C, Tietge UJ, Broedl UC et al. Dose-dependent acceleration of high-density lipoprotein catabolism by endothelial lipase. Circulation 108(17), 2121-2126 (2003).

41 Nijstad N, Wiersma H, Gautier T, van der Giet M, Maugeais C, Tietge UJ.
Scavenger receptor BI-mediated selective uptake is required for the remodelling of high density lipoprotein by endothelial lipase. J. Biol. Chem. 284(10), 6093-6100 (2009).

42 Badellino KO, Wolfe ML, Reilly MP, Rader DJ. Endothelial lipase concentrations are increased in metabolic syndrome and associated with coronary atherosclerosis. PLoS Med. 3(2), e22 (2006).

43 Khetarpal SA, Edmondson AC, Raghavan A et al. Mining the LIPG allelic spectrum reveals the contribution of rare and common regulatory variants to HDL cholesterol. PLoS Genet. 7(12), e1002393 (2011).

44 Khera AV, Cuchel M, de la Llera-Moya M et al. Cholesterol efflux capacity, high-density lipoprotein function, and atherosclerosis. N. Engl. J. Med. 364(2), 127-135 (2011).

45 Zhang CC, Kaba M, Ge G et al. Angiopoietin-like proteins stimulate ex vivo expansion of hematopoietic stem cells. Nat. Med. 12(2), 240-245 (2006).

46 Zheng J, Huynh H, Umikawa M, Silvany R, Zhang CC. Angiopoietin-like protein 3 supports the activity of hematopoietic stem cells in the bone marrow niche. Blood 117(2), 470-479 (2011).

\section{- Websites}

101 PolyPhen-2. http://genetics.bwh.harvard.edu/pph2

102 SIFT. http://sift.jcvi.org

103 Recommendations for the description of DNA sequence variants. www.hgvs.org/mutnomen/recs-DNA.html

104 Recommendations for the description of protein sequence variants. www.hgvs.org/mutnomen/recs-prot.html 